\title{
FROM PATHOPHYSIOLOGY TO MOLECULAR DIAGNOSIS IN SUDDEN UNEXPECTED DEATH IN EPILEPSY
}

\author{
Carmen-Adella Sirbu', ${ }^{1,3}$, Dan Corneci, ${ }^{1,2}$, Florentina-Cristina Plesa ${ }^{1}$, \\ Octavian-Mihai Sirbu', Anca-Maria Sandu5, Beatrice Ioan ${ }^{6}$ \\ ${ }^{1}$ Central Universitary, "Carol Davila” Emergency Military Hospital, Bucharest \\ 2"Carol Davila" University of Medicineand Pharmacy, Bucharest \\ "Titu Maiorescu" University, Bucharest \\ 4"Bagdasar-Arseni" Emergency Clinical Hospital, Bucharest \\ ${ }^{5}$ "C.I . Parhon" National I nstitute of Endocrinology, Bucharest \\ "Department of Legal Medicine, "GrigoreT. Popa” University of Medicineand Pharmacy, I asi
}

Carmen-Adella Sirbu, Dan Corneci and Beatrice loan contributed equally to this work.

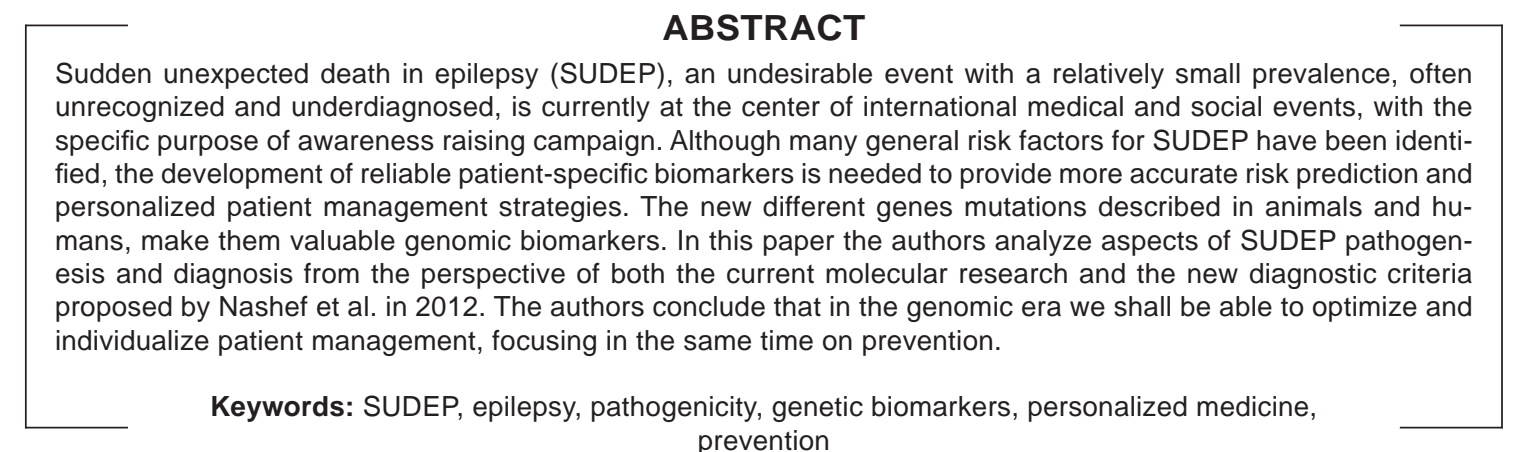
prevention

Author contributions:

Dr. Carmen Adella Sirbu: paper design, data collection, and manuscript writing.

Dr. Dan Corneci: paper design, data collection, and manuscript writing.

Dr. Beatrice loan: paper design, data collection, and manuscript writing.

Dr. Cristina -Florentina Plesa: make substantial contributions to conception and design.

Dr. Octavian Mihai Sirbu: administrative, technical support.

Dr. Anca Maria Sandu: acquisition of data, manuscript translation.

\section{INTRODUCTION}

Sudden Unexpected Death in Epilepsy (SUDEP) refers to death occurring in patients with epilepsy, without any known structural causes. The term unexpected is preferred to unexplained because more molecular etiology may be discovered in the future. An increasing number of genes are linked to SUDEP in different studies, making them good candidates for the molecular diagnostic of this condition and suggesting that the genomic biomarkers should be included in the new SUDEP diagnostic criteria.

Nashef et al. provided a unified definition of SUDEP in 2012. When defining SUDEP, a series of specific conditions has been identified, considering especially the circumstances of occurrence and diagnosis. The main features of the Definite SUDEP definition are: negative anatomopathologic examination, lack of underlying circumstances, with or without terminal seizures, excluding status epilep- 
ticus. However, because most SUDEP cases are unwitnessed, it is difficult to determine the occurrence of seizures prior to death. The second category is referred to as Definite SUDEP plus, when comorbidity could influence death. The absence of postmortem examination would include this case in Probable SUDEP. Possible SUDEP describes various circumstances, such as: a patient found dead in water - not submersed and not drowned - or a patient found dead with autopsy findings of aspiration of gastric content. Near SUDEP characterizes survivors after cardiorespiratory arrest, or deaths within days or weeks after resuscitation, without other causes of death identified during the autopsy. The sixth category is Not SUDEP, which can be defined by: death after a first assisted seizure, or with gastric contents aspiration found at autopsy, death after 5 days from resuscitation with extensive myocardial infarction documented by autopsy, patient found with neck blocked between the bed rails with signs of strangulation or dead, submersed after swimming in water (1). The definition proposed by Nashef et al. requires good medical practice guidelines comprising concrete data on toxicological sampling and analysis, genetic and immunohistochemical (IHC) tests and raising in the same time ethical and financial issues. In the same time, this new definition and classification does not mention the requirement of genetic analysis (1).

\section{PREVALENCE}

In a Danish study published in 2013, the authors found incidence prevalence of SUDEP of 41.1 per 100,000 person-years. The study design (retrospective study, based on data collected from the patients' records) suggests that the reported prevalence may have been underestimated (2). Despite these alarming epidemiological data, SUDEP is little known by physicians with patients suffering from epilepsy, as demonstrated by several studies. For example, only $33 \%$ of Canadian pediatricians have some knowledge of SUDEP and about half of those who care for children with epilepsy are familiar with the high risk of sudden death facing their patients. Most studies conclude that the risk factors for SUDEP are the following: generalized nocturnal seizures, early age of epilepsy onset, long evolution of the disease, alcohol and drugs consumption, combination of several AEDs, exposure to low temperature (3).

\section{PHYSIOPATHOLOGY}

At present, the trigger factor for SUDEP is unknown. It can be represented by respiratory dys- functions that lower the oxygen saturation, by cardiac arrhythmias as tachycardia or, more rarely, bradycardia leading to asystole, heart rate variability or postictal suppression.

Medical information about SUDEP is difficult to obtain. Most of it is provided by caregivers, witnesses, or monitoring data available when the patient dies in hospital units. According to a study published by Nashef et al., out of 26 patients with SUDEP, only 2 were assisted through death, and the retrospective analysis of the death circumstances showed that the majority died during or immediately after the seizure (4). According to Massey et al., the conditions frequently involved in SUDEP are apnea, hypoxemia, hypoventilation, pulmonary edema, and cardiac arrhythmias (5).

It seems that SUDEP could also be triggered by respiratory depression by 5-hydroxytryptamine system dysfunction (5-HT), which is incriminated in the pathogenesis of other types of sudden death as well, for example, Sudden Adult Death Syndrome (SADS) and Sudden Infant Death Syndrome (SIDS). This could also explain the depressive behavior of patients with refractory epilepsy.

The MORTEMUS retrospective study (MORTality in Epilepsy Monitoring Units Study) brings new data about SUDEP pathogenesis, highlighting postictal neuro-vegetative dysfunction as a possible causal factor in the occurrence of death. During postictal generalized EEG suppression (PGES), the risk of SUDEP increases by $1.7 \%$ per second, possibly by affecting the cardiorespiratory centers in the brainstem. In the above mentioned study, it is also shown that cardiopulmonary resuscitation (CPR) was successful when initiated within 3 minutes from onset, but it was not helpful when initiated after ten minutes (6).

On the other hand, epileptogenic discharge may affect anatomical structures that control the activity of the heart (brainstem, hypothalamus, insula, cingulate gyrus, amygdala, and autonomic centers), causing an imbalance between sympathetic and parasympathetic response, with the former predominating in most cases. Studies on animal models have shown that neurons in the hippocampus, thalamus, cerebellum and cortex are affected during long lasting seizures or status epilepticus.

Progressive neuronal loss at the level of strategic structures such as the amygdaloid nucleus or the solitary tract nucleus because of seizures, was also described. A 2015 study by a team led by Kinney shows that hippocampus disruption - either innate or acquired - affects homeostasis of circuits in the 
brainstem, including the limbic system, thus leading to cardiorespiratory instability and death (7).

Examination of a case of SUDEP requires the so-called prior verbal autopsy, whereby data can be obtained about the patient's medical condition (disease duration, type and frequency of the seizures, the recommended regimen and treatment response, comorbidities). The details on the circumstances under which the death occurred are elicited directly from witnesses when they exist, or from other sources (photographs, official police documents, medical records, etc.).

The medical examiner plays a key role in solving the cases of SUDEP, using data drawn from verbal autopsy, making the correct clinical pathological correlations and excluding other causes of death. It must be emphasized that the diagnosis of SUDEP can only be establish postmortem, with the molecular autopsy having an increasing important role. All macroscopic and microscopic details are important for including a particular case in one of the six categories proposed by Nashef: SUDEP, SUDEP plus, probable SUDEP, possible SUDEP, near SUDEP, and not SUDEP. Various discrete pathological aspects may be revealed by sectioning the brain after prior fixation. It is mandatory to examine both sides of the brain, particularly the frontal cortex and hippocampus. Microscopic brain examination of the subjects with SUDEP revealed: neuronal clusters, increased number of perivascular oligodendrocytes, gliosis, cystic gliotic lesions, decreased myelin, cerebellar Bergmann gliosis and atrophy. Autopsy results must be correlated with the results of brain MRI and EEG, for detecting possible lesions of specific brain regions.

For example, the grey matter volume in the right anterior hippocampus/amygdala and parahippocampus in sudden death cases and people at high risk, are increased when compared to controls. Also, posterior thalamic grey matter volume, involved in oxygen regulation, was reduced in these cases (8).

Other changes may also be noticed at autopsy: increased pulmonary weight, small or medium hemorrhagic pulmonary edema fibrosis of the atrioventricular node, edema in the heart conduction system, perivascular and interstitial myocardial fibrosis, reversible myocyte vacuolization and hepatic venous congestion.

It was also described postictal neurogenic stunned myocardium as a result of seizures and irreversible pathological changes under the form of interstitial and perivascular fibrosis in the sub-endocardium in four out of seven cases of SUDEP. It is possible that such changes are responsible for the increased risk of arrhythmias.

Pathological changes in the cardiac sympathetic postganglionic innervation in patients with chronic temporal lobe epilepsy have been correlated with an increased risk of SUDEP.

Toxicological analysis is important in SUDEP as it allows to assess the serum levels of anticonvulsant drugs and check the patient's adherence to treatment and, in the same time, to exclude deaths from poisoning.

Poor and non-specific autopsy results in SUDEP suggest that the molecular autopsy can provide the necessary elements to the correct diagnosis of this condition. Sodium and potassium channel dysfunctions underlie many epileptic syndromes. In the case of sodium channels, it was proved that neural isoforms are expressed in the cardiac tissue and cardiac isoforms are expressed in the nerve tissue. In 2003, Thom et al. found an elevation in hippocampal heat shock protein 70 , which is specific only to SUDEP (9).

\section{NEW GENES FOR MOLECULAR DIAGNOSIS}

The sequencing of the human genome provides us a huge benefit, from molecular medicine to human evolution. Scientists consider that genetic tests are more powerful than most existing diagnostic tests. Many new genes have been causally linked to the epilepsy and the list of the genes having a role in SUDEP is rising (10). This genetic risk will be quantified in future diagnostic and treatment guidelines and will allow for the so-called personalized medicine. Whole genome sequencing is an important tool particularly for highly heterogeneous disorders, discovering unexpected genetic mechanisms (11). Genetic conditions such as QT syndrome (LQTS) type 1, 2, 3, catecholaminergic polymorphic ventricular tachycardia (CPVT), or Dravet and Brugada syndrome were identified in several SUDEP cases. More mutations are described to date in SCN1A, SCN5A, KCNA1, KCNH2, KCNQ1, KCNE2, HTR2C, RyR2, genes, leading to increased or decreased heart rhythm, conduction blocks, which predispose to syncope and sudden death. Tester et al. studied 173 cases of sudden death (SUD) with negative postmortem examination, and identified 46 mutations of the genes mentioned above. Approximately $90-95 \%$ of sudden cardiac deaths correlate with structural changes in the heart. In addition, approximately half of the relatives of patients aged less than 50 years who died suddenly, present pathological changes of the 
channels (12). The genes that are associated with long QT syndrome are expressed throughout the body, including the heart and brain. Therefore, they are involved in the proper functioning of neurons and cardiomyocytes. In this context, we can explain the existence of the syncope expressed clinically as seizures (convulsive syncope) and the altered EEG which is identified in about $15 \%$ of patients with LQTS syndrome (13). In a study on 76 patients, Moseley found that the seizures produce the lengthening of the QT interval in $4.8 \%$ of the cases and its shortening in 3.8\% of the cases (14). More and more gene mutations are being incriminated in the development of refractory seizures, SCN8A gene being one of them. Wagon et al. introduced mutation Scn8aN1768D in a knock-in mouse, obtaining an epileptic encephalopathy model resulting in SUDEP (15).

Mutations in SCN1A and KCNA1 are largely responsible for autonomic dysfunction and, in conjunction with other mutations that lead to cardiac arrhythmias and structural defects of the heart, lead to death. Many pathological changes of the channels with a role in neuro-cardiac and neuro-respiratory modulation are expressed at the level of different anatomical structures, as previously shown. Thus, the SCN1A gene is expressed in myocytes, sinoatrial node, but also in the brain. Another example is KCNA gene expressed both in the brain and in the vagus nerve. Its inactivation using an animal model leads to epileptic seizures, cardiac arrhythmias, vagal hyperexcitability and early death (16). Tu et al. conducted a screening of the most common genes involved in long QT syndrome (SCN5A KCNQ1, KCNH2) on a number of cases of SUDEP and identified two variants with pathogenic role: SCN5A Pro1090Leu and KCNH2Arg176Trp. Future research will have to clarify whether these variants are the cause or just a risk factor for SUDEP (17). Thorough analyses of the wholeexome or whole-genome sequencing (WES, WGS) for some rare diseases bring important data for molecular diagnosis. Thus, within the WGS500 project, in which cases of refractory epilepsy were studied, Martin et al. have discovered two new genes for Ohtahara Syndrome (a form of infantile encephalopathy with refractory seizures), namely KCNT1 and PIGQ (11).

Given this new genetic tests, we can improve the prevention measures for patients with high risk of SUDEP. In addition, video EEG, ECG and oxygen saturation monitoring, representing a good medical practice, supervision by night with bed seizure monitors, breathing alarms and cardiopulmonary resuscitation training for family members can improve survival rates (3). Another measure to prevent SUDEP consists in implanting cardiac pacemakers and even defibrillators especially for the patients with high risk of SUDEP. Full cardiological evaluation of patients with epilepsy is mandatory from the onset of the disease in order to detect any rhythm and conduction abnormalities. If these are present, genotyping is necessary because gene mutations responsible for LQT interval may have different phenotypic expressions as already mentioned (epilepsy and cardiac arrhythmia)(18). For patients with sodium channelopathies, attacks treatment with channel blockers may lead to an acceptable control of the disease, which is an essential measure to prevent death. On the other hand, the physician has a duty to inform the patient of the risk of SUDEP, as well as of the preventive measures that can be established. Partemi et al. demonstrates that molecular genetic tests needs to be included for SUDEP examination and for family members screening too, because some of them could carry fatal cardiac disorders (19). The Molecular Diagnostic Core study conducted by Belmont and Goldman plays an essential role for sudden unexpected death in epilepsy research, based on genetic analysis and bioinformatics, that could ultimately lead to risk prediction in epilepsy (20).

\section{CONCLUSIONS}

Currently there are many limitations to the study of SUDEP: under-diagnosis, in/accuracy of death certificates, SUDEP, SADS, SIDS intricacies, added comorbidities, legal barriers that may block access to evidence, difficulty in obtaining consent for autopsy for research purposes or collecting blood or tissue samples for further examination, ethical considerations, financial and time constraints. Molecular autopsy is essential in SUDEP, which is a complex condition, with poor macroscopic expression, whose correct diagnosis requires corroborating data obtained through many methods. Thus, the medical guidelines should require the performance of genetic tests as a new standard of good practice in this field. In addition, future research is necessary to validate the new molecular tests for including them as new SUDEP diagnostic criteria. 


\section{REFERENCES}

1. Nashef L., So E.L., Ryvlin P., Tomson T. Unifying the definitions of sudden unexpected death in epilepsy. Epilepsia 2012, Feb; 53(2):227-33.

2. Holst A.G., Winkel B.G., Risgaard B., Nielsen J.B., Rasmussen P.V., Haunsø S., Sabers A., Uldall P., Tfelt-Hansen J. Epilepsy and risk of death and sudden unexpected death in the young: a nationwide study. Epilepsia 2013; 54(9):1613.

3. Majkowski J. Sudden Unexpected Death In Epilepsy (SUDEP) - an update. Journal of Epileptology 2013; (21): 37-54.

4. Nashef L., Garner S., Sander J.W.A.S., Fish D.R, Shorvon S.D. Circumstances of death in sudden death in epilepsy: interviews of bereaved relatives. J Neurol Neurosurg Psychiatry 1998; 64:349-352.

5. Massey C.A. et al. Mechanisms of sudden unexpected death in epilepsy: the pathway to prevention. Nat. Rev. Neurol. 2014; 10 : 271-282; published online 22 April 2014;

doi:10.1038/nrneurol.2014.64,

6. Ryvlin P. et al. Incidence and mechanisms of cardiorespiratory arrests in epilepsy monitoring units (MORTEMUS): a retrospective study. Lancet Neurol 2013; 12: 966-977.

7. Kinney H.C., Cryan J.B., Haynes R.L., Paterson D.S., et all. Dentate gyrus abnormalities in sudden unexplained death in infants: morphological marker of underlying brain vulnerability. Acta Neuropathol 2015; 129:65-80 DOI 10.1007/s00401-014-1357-0

8. Wandschneider B., Koepp M., Scott C., Micallef C., Balestrini S., Sisodiya S.M., Thom M., Harper R.M., Sander J.W., Vos S.B., Duncan J.S., Lhatoo S., Diehl B. Structural imaging biomarkers of sudden unexpected death in epilepsy. Brain. 2015 Aug 10. pii: awv233. (Epub ahead of print)

9. Thom M., Seetah S., Sisodiya S., et al. Sudden and unexpected death in epilepsy (SUDEP): evidence of acute neuronal injury using HSP-70 and C-Jun immunohistochemistry. Neuropathol Appl Neurobiol. 2003; 29:132-143.

10. Glasscock E. Genomic biomarkers of SUDEP in brain and heart. Epilepsy Behav. 2014 Sep; 38:172-9. doi: 10.1016/j. yebeh.2013.09.019. Epub 2013 Oct 17

11. Martin H.C., Kim G.E., Pagnamenta A.T., Murakami Y., Carvill G.L., Meyer E., Copley R.R. et all. Clinical whole-genome sequencing in severe early-onset epilepsy reveals new genes and improves molecular diagnosis. Hum Mol Genet. 2014 Jun 15; 23(12):3200-11. doi: 10.1093/hmg/ddu030. Epub 2014 Jan 25.

12. Tester D.J., Medeiros-Domingo A., Will M.L., Haglund C.M., Ackerman M.J. Cardiac Channel Molecular Autopsy: Insights From 173 Consecutive Cases of Autopsy-Negative Sudden Unexplained Death Referred for Postmortem Genetic Testing. Mayo Clinic Proceedings, june 2012; 87, 6: 524-539.

13. Anderson J.H., Bos J.M., Cascino G.D., Ackerman M.J. Prevalence and spectrum of electroencephalogram-identified epileptiform activity among patients with long QT syndrome. Heart Rhythm 2014; 11: 53-57.

14. Moseley B.D. et al. Electrocardiographic and oximetric changes during partial complex and generalized seizures. Epilepsy Res. 2011; 95, 237-245.

15. Wagnon J.L., Korn M.J., Parent R., Tarpey T.A., Jones J.M. et all. Convulsive seizures and SUDEP in a mouse model of SCN8A epileptic encephalopathy. Human Molecular Genetics, 2015, Vol. 24, No. 2:506-515, doi:10.1093/hmg/ddu470.

16. Kalume F., Westenbroek R.E., Cheah C.S., et al. Sudden unexpected death in a mouse model of Dravet syndrome. J Clin Invest 2013; 123:1798-1808.

17. Tu E., Bagnall R.D., Duflou J., Semsarian C. Post-Mortem Review and Genetic Analysis of Sudden Unexpected Death in Epilepsy (SUDEP) Cases. Brain pathology 2010, doi:10.1111/j.1750-3639.2010.00438.

18. Eastaugh A.J., Thompson T., Vohra J.K., O'Brien T.J., Winship I. Sudden unexpected death, epilepsy and familial cardiac pathology. J Clin Neurosci. 2015 Oct; 22(10):1594-600. doi: 10.1016/j.jocn.2015.05.002. Epub 2015 Jul 17.

19. Partemi S., Vidal M.C., Striano P., Campuzano O., Allegue C., Pezzella M., et al. Genetic and forensic implications in epilepsy and cardiac arrhythmias: a case series. Int J Legal Med. 2015 May; 129(3):495-504. doi: 10.1007/s00414-014-1063-4. Epub 2014 Aug 15.

20. Lhatoo S., Noebels J., Whittemore V. The NINDS Center for SUDEP Research, Sudden unexpected death in epilepsy: Identifying risk and preventing mortality, Epilepsia, 56(11):1700-1706, 2015, doi: 10.1111/epi.13134 